\title{
AIAA 2003-5204
}

Quasi-One-Dimensional Modeling of Pulse Detonation Rocket Engines

C. I. Morris

NASA/George C. Marshall Space Flight Center TD40/Propulsion Research Center

Marshall Space Flight Center, AL USA 35812

39th AIAA/ASME/SAE/ASEE Joint Propulsion Conference \& Exhibit July 20-23, 2003/Huntsville, AL 


\title{
Quasi-One-Dimensional Modeling of Pulse Detonation Rocket Engines
}

\author{
C. I. Morris* \\ NASA/George C. Marshall Space Flight Center \\ TD40/Propulsion Research Center \\ Marshall Space Flight Center, AL USA 35812
}

\begin{abstract}
Pulse detonation rocket engines (PDREs) offer potential performance improvements over conventional designs, but represent a challenging modeling task. A quasi 1-D, finite-rate chemistry CFD model for a PDRE is described and implemented. A parametric study of the effect of blowdown pressure ratio on the performance of an optimized, fixed PDRE nozzle configuration is reported. The results are compared to a steady-state rocket system using similar modeling assumptions.
\end{abstract}

\section{Introduction}

$\mathbf{P}$ ULSED detonation rocket engines (PDREs) have generated considerable research interest in recent years ${ }^{1}$ as a chemical propulsion system potentially offering improved performance and reduced complexity compared to conventional rocket engines. The detonative mode of combustion employed by these devices offers a thermodynamic advantage over the constant-pressure deflagrative combustion mode used in conventional rocket engines and gas turbines. However, while this theoretical advantage has spurred a great deal of interest in building PDRE devices, the unsteady blowdown process intrinsic to the PDRE has made realistic estimates of the actual propulsive performance problematic. The recent review article by Kailasanath ${ }^{2}$ highlights some of the difficulties in comparing the available experimental measurements with numerical models.

In a previous paper by the author, ${ }^{3}$ parametric studies of the performance of a single, straight-tube PDRE were reported. A 1-D, unsteady method of characteristics code, employing a constant- $\gamma$ assumption behind the detonation front, was developed for that study. Models of this type are computationally inexpensive, and are particularly useful for parametric performance comparisons. For example, a plot showing the specific impulse of various PDRE and steady-state rocket engine (SSRE) configurations as a function of blowdown pressure ratio $\left(\mathrm{P}_{\text {initial }} / \mathrm{P}_{\text {final }}\right)$ is shown in Figure 1. Note that the SSRE performance calculations employ the same constant $\gamma$ assumption used in the PDRE calculations. The performance curves indicate that a straight-tube PDRE provides superior specific impulse, compared to a SSRE with a sonic nozzle, over the entire range of pressure ratios. Note, however, that a straighttube PDRE in general does not compare favorably to a SSRE fitted with an optimized converging-diverging (C-D) supersonic nozzle, particularly at the high pressure ratios typical for boost or in-space rocket applications. This result

\footnotetext{
* Research Engineer, Member AIAA.

This paper is a work of the U.S. Government and is not subject to copyright protection in the United States.
}

is largely due to the choked outflow from a straight-tube PDRE. However, calculations of an ideal expansion of the PDRE outflow show that if a dynamically optimized supersonic nozzle could be fitted to a PDRE, then the specific impulse of the device would exceed that of a comparable SSRE. While such a nozzle is a considerable idealization, it is clear that nozzle design and optimization will play a critical role in whether the performance potential of PDREs can be effectively realized in practice.

The purpose of this paper is to report efforts to study the effect of nozzles on PDRE gasdynamics and performance. Details of the quasi 1-D, finite-rate chemistry CFD model developed by the author are provided first. A parametric study of the effect of blowdown pressure ratio on the performance of an optimized, fixed PDRE nozzle configuration is then reported. The results are then compared to a SSRE system using similar modeling assumptions.

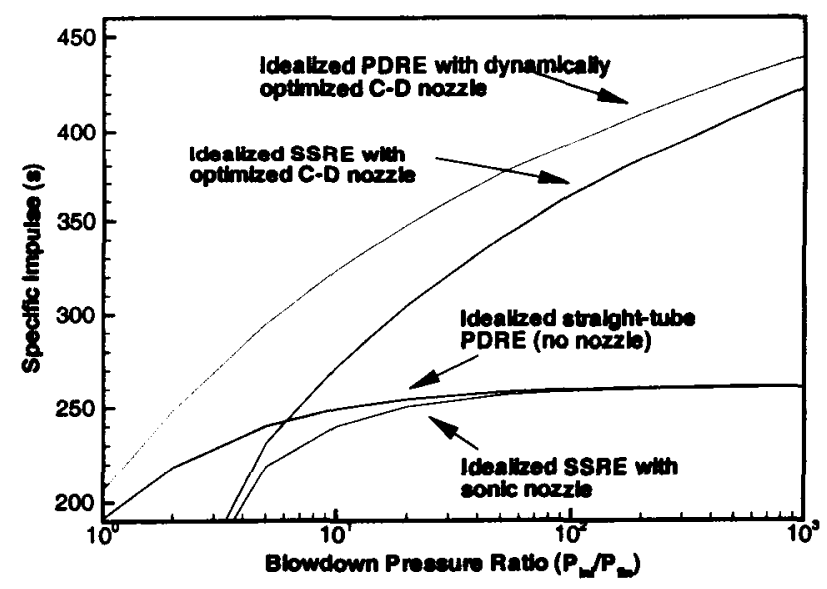

Fig. 1 Performance comparison of a pulse detonation rocket engine (PDRE) with a conventional steady-state rocket engine (SSRE) equipped with both sonic and optimized supersonic converging-diverging nozzles. The specific heat ratio, $\gamma$, is held constant in all models. The final blowdown pressure at the end-wall in the PDRE is equal to the ambient pressure $\left(\mathbf{P}_{\text {final }}=\mathbf{P}_{\text {ambient }}\right)$. Propellant mixture: stoichiometric $\mathrm{H}_{2} \cdot \mathrm{O}_{2}$. Propellant initial conditions: $P_{\text {initial }}=1 \mathrm{~atm}, T_{\text {inittal }}=300 \mathrm{~K}$. 


\section{Governing Equations}

The quasi-one-dimensional Euler equations are the governing model used in this study. A quasi-1-D model is at best an approximation of a pulsed detonation or steadystate rocket engine. Real detonation waves exhibit clear multi-dimensional behavior. Additionally, flow separation in rocket engine nozzles is strongly dependent on multidimensional and viscous effects. However, as the primary goal of this study is to determine the effect of nozzles on pulse detonation rocket systems to first order, the quasi-1$D$ Euler equations were deemed a reasonable model. The time-dependent form of these equations, including the effect of finite-rate chemistry, is written as follows:

$$
\frac{\partial \mathbf{U}}{\partial t}+\frac{1}{S} \frac{\partial \mathbf{F S}}{\partial x}=\mathbf{H}+\mathbf{W}
$$

where $S$ represents the cross sectional area of the duct, and is a function of distance, $\mathbf{x}$. The state vector, $\mathbf{U}$, and convective flux vector, $\mathbf{F}$, are given by

$$
\mathbf{U}=\left[\begin{array}{c}
\rho_{1} \\
\rho_{2} \\
\vdots \\
\rho_{n s} \\
\rho u \\
\rho e
\end{array}\right], \quad \mathbf{F}=\left[\begin{array}{c}
\rho_{1} u \\
\rho_{2} u \\
\vdots \\
\rho_{n s} u \\
\rho u^{2}+p \\
u(\rho e+p)
\end{array}\right]
$$

The source vectors accounting for the effect of area change, $\mathbf{H}$, and for the effect of finite-rate chemistry, $\mathbf{W}$, are

$$
\mathbf{H}=\left[\begin{array}{c}
0 \\
0 \\
\vdots \\
0 \\
\frac{p}{S} \frac{\partial S}{\partial x} \\
0
\end{array}\right], \quad \mathbf{W}=\left[\begin{array}{c}
w_{1} \\
w_{2} \\
\vdots \\
w_{n s} \\
0 \\
0
\end{array}\right]
$$

Here, $\rho_{1}, \ldots, \rho_{n s}$ represent the mass densities of the $n s$ individual chemical species in the problem. The total gas density $\rho$ is sum of the individual species densities, $\rho=$ $\sum_{k=1}^{n s} \rho_{k} . u$ is the gas velocity in the $\mathrm{x}$ direction. $p$ is the gas pressure determined by the ideal gas law,

$$
p=\sum_{k=1}^{n s} \frac{\rho_{k}}{M_{k}} R T
$$

where $M_{k}$ is the molecular weight of the species, $R$ is the universal gas constant, and $T$ is the gas temperature. $e$ is the total energy per unit mass of the gas, $e=\epsilon+\frac{1}{2} u^{2}$, where $\epsilon$ is the specific energy per unit mass, and for the thermally perfect gas model considered here $\epsilon$ is a function of temperature and gas composition,

$$
\epsilon=\sum_{k=1}^{n s} Y_{k} \epsilon_{k}(T)
$$

where $Y_{k}$ represents the mass fraction of species $k, Y_{k}=$ $\rho_{k} / \rho$, and $\epsilon_{k}(T)$ is the specific energy per unit mass of species $k$, as a function of temperature. In this work, the ideal gas thermodynamic fits of McBride et al. ${ }^{4}$ are used to calculate the energy, specific heat, and Gibbs free energy for the 9 species in the problem $\left(\mathrm{N}_{2}, \mathrm{O}_{2}, \mathrm{H}_{2}, \mathrm{OH}, \mathrm{H}_{2} \mathrm{O}, \mathrm{H}\right.$, $\mathrm{O}, \mathrm{HO}_{2}$, and $\mathrm{H}_{2} \mathrm{O}_{2}$ are included).

\section{Chemistry Model}

The source terms for finite-rate chemistry, $w_{k}$ in Eq. 3, are calculated from the sum of relevant chemical reactions for each species. Thus, for a chemical mechanism of $n r$ elementary reactions, each reaction is expressed as

$$
\sum_{l=1}^{n s} v_{l, n}^{\prime} X_{l} \rightleftharpoons \sum_{l=1}^{n s} v_{l, n}^{\prime \prime} X_{l}
$$

where the $v_{l, n}^{\prime}$ are the stoichiometric coefficients of species $l$ on the reactant side of reaction $n$, while the $v_{l, n}^{\prime \prime}$ are the corresponding stoichiometric coefficients on the product side. The production term for each species may be written as

$$
\begin{aligned}
w_{k} \equiv & \frac{\partial \rho_{k}}{\partial t}=M_{k} \sum_{n=1}^{n r}\left(v_{k, n}^{\prime \prime}-v_{k, n}^{\prime}\right) \\
& \cdot\left[k_{f} \prod_{l=1}^{n . s}\left(\frac{\rho_{l}}{M_{l}}\right)^{v_{l, n}^{\prime}}-k_{b} \prod_{l=1}^{n s}\left(\frac{\rho_{l}}{M_{l}}\right)^{v_{l, n}^{\prime \prime}}\right]
\end{aligned}
$$

and $k_{f}$ and $k_{b}$ represent the forward and backward reaction rates, respectively. Details of the chemical kinetics mechanism for $\mathrm{H}_{2}-\mathrm{O}_{2}$ combustion used in this work are shown in Table 1. This mechanism was developed by Petersen and Hanson ${ }^{5}$ to model $\mathrm{H}_{2}-\mathrm{O}_{2}$ ignition at the elevated pressures typical of practical high-speed propulsion systems. The rate coefficients for reactions without pressure dependence take on the conventional Arrhenius form:

$$
k_{f}(T)=A T^{n} \exp \left(-E_{\mathrm{act}} / R T\right)
$$

where $A$ is a constant, and $E_{\text {act }}$ is the activation energy in $\mathrm{cal} / \mathrm{mol}$. All reactions in the mechanism are reversible. The reverse reaction rate, $k_{b}$, is calculated form the forward rate (Eq. 8) and the equilibrium constant.

Pressure-dependent reactions are modeled using the approach given by Kee: ${ }^{6}$

$$
k_{f}=k_{\infty}\left[P_{r} /\left(1+P_{r}\right)\right] F
$$

where the reduced pressure, $P_{r}$, is

$$
P_{r}=\frac{k_{0}[\mathrm{M}]}{k_{\infty}}
$$

and the correction factor, $F$, is in the Troe ${ }^{7}$ form:

$$
\ln F=\left\{1+\frac{\ln P_{r}+c}{n-d\left(\ln P_{r}+c\right)}\right\}^{-1} \ln F_{c}
$$

The Troe centering parameter, $F_{c}$ is given by

$$
\begin{aligned}
F_{c}= & (1-a) \exp \left(-T / T^{* * *}\right)+a \exp \left(-T / T^{*}\right)+ \\
& \exp \left(-T^{* *} / T\right)
\end{aligned}
$$

where the constants $c=-0.4-0.67 \ln F_{r}, n=-0.75-$ $1.27 \ln F_{c}$, and $d=0.14$. 
Table 1 Chemical kinetics mechanism for $\mathrm{H}_{2}-\mathrm{O}_{2}$ combustion, from Petersen and Hanson. 5

\begin{tabular}{|c|c|c|c|c|c|}
\hline Number & Reaction $^{a}$ & & $A^{b}$ & $n^{b}$ & $E_{\text {act. }}^{b}$ \\
\hline $\mathbf{1}$ & $\mathrm{O}+\mathrm{H}_{2} \rightleftharpoons \mathrm{H}+\mathrm{OH}$ & & $5.00 \times 10^{6}$ & 2.70 & 6290 \\
\hline $2^{c}$ & $\mathrm{H}+\mathrm{O}_{2}+\mathrm{M} \rightleftharpoons \mathrm{HO}_{2}+\mathrm{M}$ & & $2.80 \times 10^{18}$ & -0.90 & 0 \\
\hline 3 & $\mathrm{H}+\mathrm{O}_{2}+\mathrm{O}_{2} \rightleftharpoons \mathrm{HO}_{2}+\mathrm{O}_{2}$ & & $3.00 \times 10^{20}$ & -1.70 & 0 \\
\hline 4 & $\mathrm{H}+\mathrm{O}_{2}+\mathrm{H}_{2} \mathrm{O} \rightleftharpoons \mathrm{HO}_{2}+\mathrm{H}_{2} \mathrm{O}$ & & $9.38 \times 10^{18}$ & -0.80 & 0 \\
\hline 5 & $\mathrm{H}+\mathrm{O}_{2}+\mathrm{N}_{2} \rightleftharpoons \mathrm{HO}_{2}+\mathrm{N}_{2}$ & & $2.60 \times 10^{19}$ & -1.20 & 0 \\
\hline 6 & $\mathrm{H}+\mathrm{O}_{2} \rightleftharpoons \mathrm{O}+\mathrm{OH}$ & & $8.30 \times 10^{13}$ & 0.00 & 14413 \\
\hline 7 & $\mathrm{H}+\mathrm{HO}_{2} \rightleftharpoons \mathrm{O}_{2}+\mathrm{H}_{2}$ & & $2.80 \times 10^{13}$ & 0.00 & 1068 \\
\hline 8 & $\mathrm{H}+\mathrm{HO}_{2} \rightleftharpoons \mathrm{OH}+\mathrm{OH}$ & & $1.34 \times 10^{14}$ & 0.00 & 635 \\
\hline 9 & $\mathrm{H}+\mathrm{H}_{2} \mathrm{O}_{2} \rightleftharpoons \mathrm{HO}_{2}+\mathrm{H}_{2}$ & & $1.21 \times 10^{7}$ & 2.00 & 5200 \\
\hline 10 & $\mathrm{OH}+\mathrm{H}_{2} \rightleftharpoons \mathrm{H}_{2} \mathrm{O}+\mathrm{H}$ & & $2.16 \times 10^{8}$ & 1.50 & 5200 \\
\hline \multirow[t]{2}{*}{$11^{d, e}$} & $\mathrm{OH}+\mathrm{OH}+\mathrm{M} \rightleftharpoons \mathrm{H}_{2} \mathrm{O}_{2}+\mathrm{M}$ & $k_{\infty}$ & $7.40 \times 10^{13}$ & -0.40 & 0 \\
\hline & & $k_{0}$ & $2.30 \times 10^{18}$ & -0.90 & -1700 \\
\hline 12 & $\mathrm{OH}+\mathrm{HO}_{2} \rightleftharpoons \mathrm{O}_{2}+\mathrm{H}_{2} \mathrm{O}$ & & $2.90 \times 10^{13}$ & 0.00 & -500 \\
\hline \multirow[t]{2}{*}{$13^{f}$} & $\mathrm{OH}+\mathrm{H}_{2} \mathrm{O}_{2} \rightleftharpoons \mathrm{HO}_{2}+\mathrm{H}_{2} \mathrm{O}$ & $k_{a}$ & $1.75 \times 10^{12}$ & 0.00 & 320 \\
\hline & & $k_{b}$ & $5.80 \times 10^{14}$ & 0.00 & 9560 \\
\hline \multirow[t]{2}{*}{$14^{g}$} & $\mathrm{HO}_{2}+\mathrm{HO}_{2} \rightleftharpoons \mathrm{O}_{2}+\mathrm{H}_{2} \mathrm{O}_{2}$ & $k_{c}$ & $1.30 \times 10^{11}$ & 0.00 & -1630 \\
\hline & & $k_{d}$ & $4.20 \times 10^{14}$ & 0.00 & 12000 \\
\hline $15^{h}$ & $\mathrm{O}+\mathrm{O}+\mathrm{M} \rightleftharpoons \mathrm{O}_{2}+\mathrm{M}$ & & $1.20 \times 10^{17}$ & -1.00 & 0 \\
\hline $16^{d}$ & $\mathrm{O}+\mathrm{H}+\mathrm{M} \rightleftharpoons \mathrm{OH}+\mathrm{M}$ & & $5.00 \times 10^{17}$ & -1.00 & 0 \\
\hline $17^{i}$ & $\mathrm{H}+\mathrm{OH}+\mathrm{M} \rightleftharpoons \mathrm{H}_{2} \mathrm{O}+\mathrm{M}$ & & $2.20 \times 10^{22}$ & -2.00 & 0 \\
\hline $18^{j}$ & $\mathrm{H}+\mathrm{H}+\mathrm{M} \rightleftharpoons \mathrm{H}_{2}+\mathrm{M}$ & & $1.00 \times 10^{18}$ & -1.00 & 0 \\
\hline
\end{tabular}

Note: species are $\mathrm{N}_{2}, \mathrm{O}_{2}, \mathrm{H}_{2}, \mathrm{H}_{2} \mathrm{O}, \mathrm{OH}, \mathrm{H}, \mathrm{O}, \mathrm{HO}_{2}, \mathrm{H}_{2} \mathrm{O}_{2}$.

${ }^{a}$ All reactions are reversible.

$b_{k_{f}}(T)=A T^{n} \exp \left(-E_{\text {act }} / R T\right)$; units are in cal, $\mathrm{mol}, \mathrm{cm}^{3}$, and s.

${ }^{c} \mathrm{M}$ does not include $\mathrm{O}_{2}, \mathrm{H}_{2} \mathrm{O}$, or $\mathrm{N}_{2} ;$ all collision efficiencies $=1.0$.

${ }^{d}$ Collision efficiencies for $\mathrm{M} ; \mathrm{H}_{2}=2.0, \mathrm{H}_{2} \mathrm{O}=6.0$, all others $=1.0$.

'Troe parameters: $a=0.7346, T^{* * *}=94, T^{*}=1756, T^{* *}=5182$.

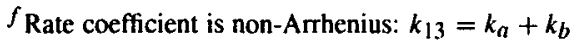

${ }$ Rate coefficient is non-Arrhenius: $k_{14}=k_{c}+k_{d}$

${ }^{h}$ Collision efficiencies for $\mathrm{M} ; \mathrm{H}_{2}=2.4, \mathrm{H}_{2} \mathrm{O}=15.4$, all others $=1.0$.

${ }^{i}$ Collision efficiencies for $\mathrm{M} ; \mathrm{H}_{2}=0.73, \mathrm{H}_{2} \mathrm{O}=3.65$, all others $=1.0$.

$j$ Collision efficiencies for $M ; H_{2}=1.7, \mathrm{H}_{2} \mathrm{O}=7.0$, all others $=1.0$.

\section{Numerical Method}

The governing quasi 1-D Euler equations are solved in finite-volume form throughout the entire domain. The equations are solved in split form, in which a fluid convection subroutine solves Eq. 1 without the chemistry source vector $\mathbf{W}$,

$$
\frac{\partial \mathbf{U}}{\partial t}+\frac{1}{S} \frac{\partial \mathbf{F S}}{\partial x}=\mathbf{H}
$$

and a finite-rate chemistry integration subroutine solves Eq. 1 as a system of ordinary differential equations ignoring $\mathbf{F}$ and $\mathbf{H}$,

$$
\frac{\partial \mathrm{U}}{\partial t}=\mathbf{W}
$$

The code utilizes the Strang ${ }^{8}$ second-order time step splitting approach to couple the fluid convection and finiterate chemistry subroutines. For each complete time step, the chemistry subroutine is first called for one-half of a time step, followed by a full fluid convection time step, and then followed by another half-step of the chemistry routine. Thus, in operator notation, the solution $\mathbf{U}$ at time-step $n+1$ is

$$
\mathrm{U}^{n+1}=\mathcal{L}_{r}^{\Delta t / 2} \mathcal{L}_{f}^{\Delta t} \mathcal{L}_{r}^{\Delta t / 2} \mathbf{U}^{n}
$$

where $\mathcal{L}_{f}$ represents the fluid subroutine solving Eq. 13 and $\mathcal{L}_{c}$ represents the finite-rate chemistry subroutine solving Eq. 14. The time step splitting approach allows both high-quality fluid and chemical solvers to be developed and tested independently, and then joined together in relatively straightforward fashion. As described by Oran and Boris, ${ }^{9}$ time-step splitting works well when relatively small timesteps are used. Since the global time-step in the present code is strictly limited by the explicit Courant-FriedrichsLewy (CFL) condition, this is not thought to be a problem. 
The fluid solver used here is the explicit, 2nd-order accurate (in time and space), symmetric-TVD algorithm described by Yee. ${ }^{10}$ The solver employs Roe's approximate Riemann solver modified for nonequilibrium ideal gases. ${ }^{11}$ It also incorporates suggestions by Larrouturou ${ }^{12}$ to ensure species positivity.

As with most reactive flow problems, the timeintegration of the chemistry mechanism in this work requires a stiff-ODE solver to ensure accuracy. The method used here employs a semi-implicit trapezoidal scheme as a predictor,

$$
\left[\mathbf{I}-\frac{1}{2} \Delta t \mathbf{J}\right]\left(\mathbf{U}_{p}^{n+1}-\mathbf{U}^{n}\right)=\Delta t \mathbf{W}^{n}
$$

where $\mathbf{I}$ is the identity matrix, $\mathbf{J}$ is the Jacobian of $\mathbf{W}, \mathbf{J}=$ $\partial \mathbf{W} / \partial \mathbf{U}$, and $\mathbf{U}_{p}^{n+1}$ is the predicted next value of $\mathbf{U}$. Newton iteration of the trapezoidal scheme is used as a corrector,

$$
\begin{aligned}
& {\left[\mathbf{I}-\frac{1}{2} \Delta t \mathbf{J}\right]\left(\mathbf{U}_{c}^{n+1}-\mathbf{U}_{p}^{n+1}\right)=} \\
& {\left[\mathbf{U}^{n}-\mathbf{U}_{p}^{n+1}+\frac{1}{2} \Delta t\left(\mathbf{W}^{n}+\mathbf{W}_{p}^{n+1}\right)\right]}
\end{aligned}
$$

where $\mathbf{U}_{c}^{n+1}$ is the corrected next value of $\mathbf{U}$.

The chemistry subroutine is designed to use several local time steps, if necessary for accuracy, within a given global half-step. The relative magnitude of the corrector $\left(\mathbf{U}_{c}^{n+1}-\mathbf{U}_{p}^{n+1}\right) / \mathbf{U}_{r}^{n+1}$ is used as basis for adjusting the local time step, similar to the accuracy-based time step procedure described by Mott. ${ }^{13}$ The Jacobian $\mathbf{J}$ is evaluated analytically once per local time step at $\mathbf{U}^{n}$. Thus, the LU decomposition of the bracketed matrix terms on the left hand side of Eq. 16 can be re-used in Eq. 17.

\section{PDRE Simulations}

The PDRE system studied here (Fig.2) is highly idealized, consisting of a constant-area $(2.0 \mathrm{~cm}$ diameter $)$ detonation tube $16 \mathrm{~cm}$ in length. One end is closed and the other end open to the environment, or attached to a simple conical converging-diverging nozzle section. The nozzle initially converges at a constant $14^{\circ}$ angle to a throat $1.8 \mathrm{~cm}$ in diameter, and then diverges again at a constant $14^{\circ}$ to a final exit diameter specified by the user. The computational domain automatically scales to fit the nozzle geometry. The detonation tube is pre-filled with a gaseous propellant mixture with no initial velocity. Stoichiometric $\mathrm{H}_{2}-\mathrm{O}_{2}$ at an initial pressure of $1 \mathrm{~atm}$, and initial temperature of $300 \mathrm{~K}$ is utilized for all calculations in this work. An idealized, massless diaphragm isolates the propellant mixture from the nozzle and ambient environment until ruptured by the detonation wave. The nozzle section is initially filled with $\mathrm{H}_{2}$ gas at the specified ambient pressure.

The detonation in each simulation is initiated by specifying equilibrium combustion conditions (computed at constant $\rho$ and $\epsilon$ ) in the cell adjacent to the closed end-wall.

Ghost cells are utilized to specify the boundary conditions in the problem. A reflection-type boundary condition

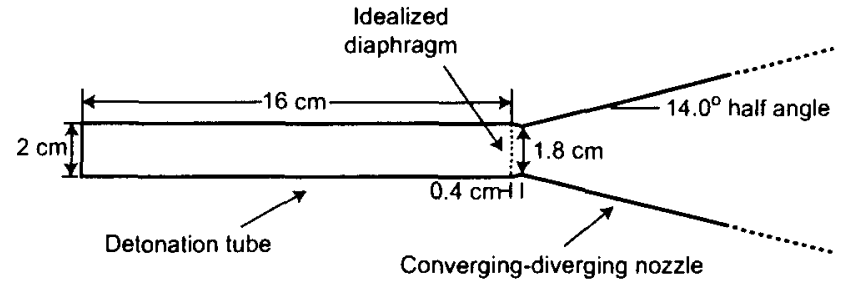

Fig. 2 Schematic of PDRE geometry studied.

is utilized at the closed end of the detonation tube to simulate a solid wall. The method of characteristics ${ }^{14}$ is used to calculate the exit flow boundary condition. For sonic (choked) or supersonic exit flow, all exit flow properties are determined by the interior flow. For subsonic exit flow, the ambient pressure is specified and the method of characteristics is used to compute the remaining flow properties. If reverse flow is detected at the exit, a subsonic inflow boundary condition is calculated using the ambient $\mathrm{H}_{2}$ gas as a constant pressure and enthalpy reservoir.

Two additional special restrictions are imposed in the simulations. In order to simulate the effect of an idealized diaphragm, only the detonation tube portion of the domain (from the closed wall to the diaphragm location) is computed initially. A reflection-type boundary condition is specified at the diaphragm location until the pressure in the adjacent cell rises $1.0 \%$ above the initial fill value. This special restriction is subsequently removed, and the entire domain is computed. Additionally, there is a check performed when the exit flow is supersonic. Since in this case the exit boundary conditions are entirely calculated from the interior flow, there is no way for the exit flow to return to a subsonic condition. ${ }^{15}$ Therefore, at each time step a check is made to determine if the pressure from standing normal shock at the exit is less than the ambient pressure. If true, then the normal shock properties are specified in the last interior cell, and a subsonic outflow boundary condition computed at the exit.

\section{Grid Resolution}

Grid resolution is a critical issue in all finite-rate chemistry CFD models. Due to the extremely fast kinetics of the $\mathrm{H}_{2}-\mathrm{O}_{2}$ system, numerical modeling of the reaction zone of undiluted $\mathrm{H}_{2}-\mathrm{O}_{2}$ Chapman-Jouguet (C-J) detonations requires an exceptionally fine grid spacing. The $\mathrm{C}$-J detonation velocity of stoichiometric $\mathrm{H}_{2}-\mathrm{O}_{2}$ at and initial condition of $1 \mathrm{~atm}$ and $300 \mathrm{~K}$ is calculated by the NASA CEA thermochemical code ${ }^{16}$ to be $2837.1 \mathrm{~m} / \mathrm{s}$. Using the calculated frozen shock properties at this velocity, and the chemical kinetics mechanism described previously, the length of the C-J reaction zone for this mixture can be estimated as $\sim 0.02 \mathrm{~mm}$. Thus, a grid spacing of roughly $1 \mu \mathrm{m}$ would be required to resolve the reaction zone. This is not currently practical, nor is it necessary for the performance estimates of this study. The chief requirement here is that the grid resolution accurately calculate the C-J state and velocity.

A grid resolution comparison of pressure and tempera- 

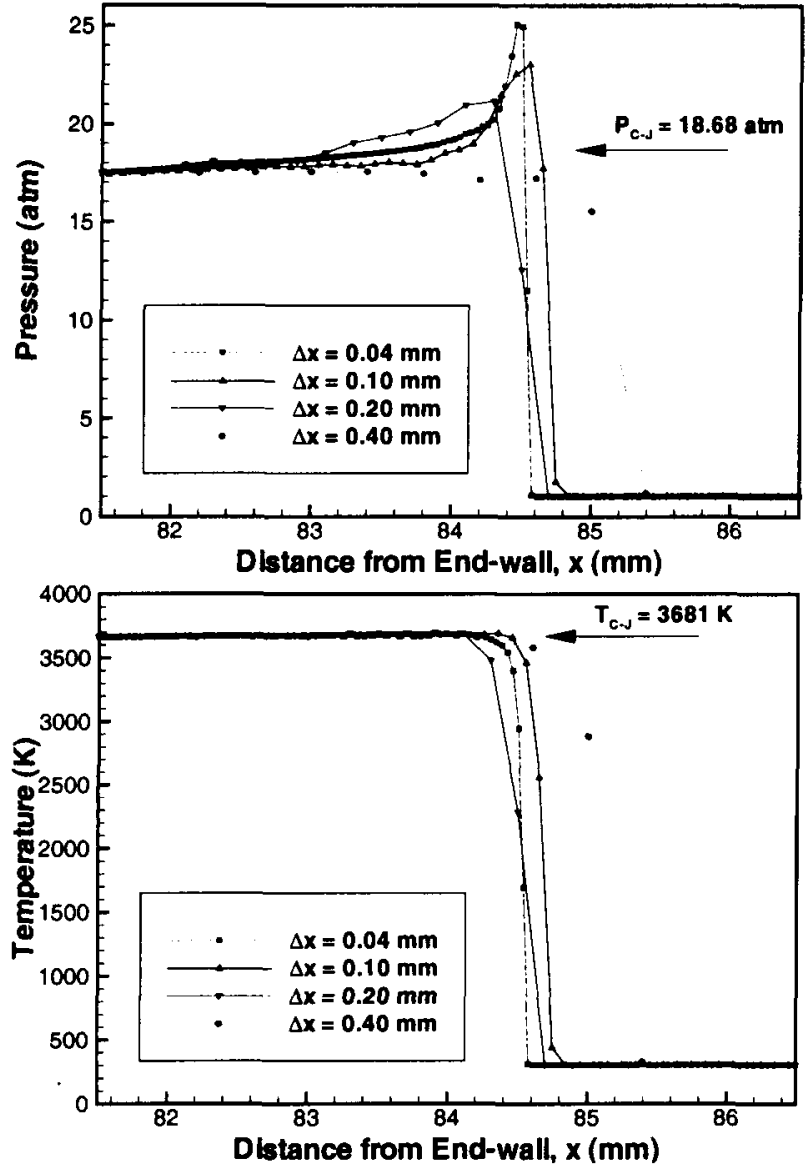

Fig. 3 Grid resolution comparison showing pressure (upper panel) and temperature (lower panel) profiles of a propagating $\mathrm{C}-\mathrm{J}$ detonation wave at $t=30 \mu$ s after initiation.

ture profiles of a propagating C-J detonation, $t=30 \mu \mathrm{s}$ after initiation, is shown in Fig. 3. The resolutions vary over one order of magnitude. As even the finest resolution here, $\Delta x=0.04 \mathrm{~mm}$, is twice the estimated reaction zone size, none of the resolutions can accurately capture the ignition process behind the leading shock wave. Rather, in all cases, the propagating $\mathrm{C}-\mathrm{J}$ detonation is smeared over several cells, resulting in the pressure spike and subsequent relaxation toward the C-J state in the finer grids. All four resolutions capture the detonation velocity with reasonable accuracy $(\sim 2836 \mathrm{~m} / \mathrm{s}$ for $\Delta \mathrm{x}=0.04 \mathrm{~mm}, \sim 2840 \mathrm{~m} / \mathrm{s}$ for the other resolutions), though the precision of this estimate is naturally lower for the coarser grids. Note, however, that in two finer grids the pressure relaxes to the $\mathrm{C}-\mathrm{J}$ value in a significantly shorter distance than in the coarser grids. Based on these observations, a uniform grid spacing of $\Delta x$ $=0.10 \mathrm{~mm}$ was utilized for all simulations in this study.

\section{Thrust Calculations}

The time-dependent thrust is calculated at each time step by two different methods. One measure of the thrust assumes a control volume tightly bounding the solid surfaces of the PDRE, and is determined by integrating the pressure difference across all surfaces. A second measure assumes a rectangular control volume encapsulating the PDRE. This measure of thrust is determined from the sum of the time rate of change of the internal momentum integral across the domain, the momentum flux from the nozzle section, and the pressure difference across the control volume. In general, there is excellent agreement between the two thrust calculations, and the time-integrated impulse calculations agree to within $0.1 \%$. In all simulations, the calculation proceeds until the pressure at the closed end-wall is equal to the ambient pressure. Thus, these simulations should be thought of as single-shot results.

\section{Results and Discussion}

The blowdown pressure ratio is one of the most critical factors governing the performance of any rocket-type system. In this study, this parameter is defined as the ratio of the initial fill pressure in the detonation tube to the ambient pressure. The quasi 1-D CFD model is used to determine the optimum expansion ratio, $\varepsilon$, for a converging-diverging nozzle for a range of blowdown pressure ratios ranging from 1-1000. At each pressure ratio, a variety of expansion ratios were tested, and a manual search was performed to determine the optimum expansion ratio. In each case, the optimum expansion ratio corresponds to the maximum total impulse. Two example cases will be examined in detail, followed by a comparison of the results among several systems over the entire pressure range.

\section{PDRE Thrust and Pressure Histories}

The optimum nozzle exit diameter for the model PDRE system at a blowdown pressure ratio of 1 is found to be $2.2 \mathrm{~cm}$. This corresponds to an expansion ratio of 1.49 . Since the manual search for optimal exit radius was conducted in $1 \mathrm{~mm}$ increments, there is a variance on this expansion ratio of \pm 0.26 . The mixture-based specific impulse of the optimized C-D system is $197.48 \mathrm{~s}$. This is a modest improvement over the $192.19 \mathrm{~s}$ provided by the baseline detonation tube without any nozzle at this pressure ratio. A comparison between the single-shot thrust history for the optimized converging-diverging nozzle and the baseline detonation tube is shown in the upper panel of Fig. 4. The corresponding exit pressure history is shown in the lower panel of the figure. It is evident from the thrust history that the reduced diameter of the throat in the C$\mathrm{D}$ system increases the overall blowdown time compared to the baseline detonation tube. Additionally, the thrust for the optimized C-D system is actually lower than that of the baseline detonation tube for a significant portion of the early blowdown history. The main benefit of the C-D nozzle is derived from later in the blowdown history when the supersonic exit flow from the nozzle provides slightly greater thrust than the choked flow of the baseline tube. Note that flow from the C-D nozzle becomes overexpanded late in the blowdown history, and eventually a normal shock forms at the exit by the procedure described in the previous section. There is even a small period of negative thrust at the very end of the history, as shown by the peak impulse marker in the upper panel of the figure. It is evident that 

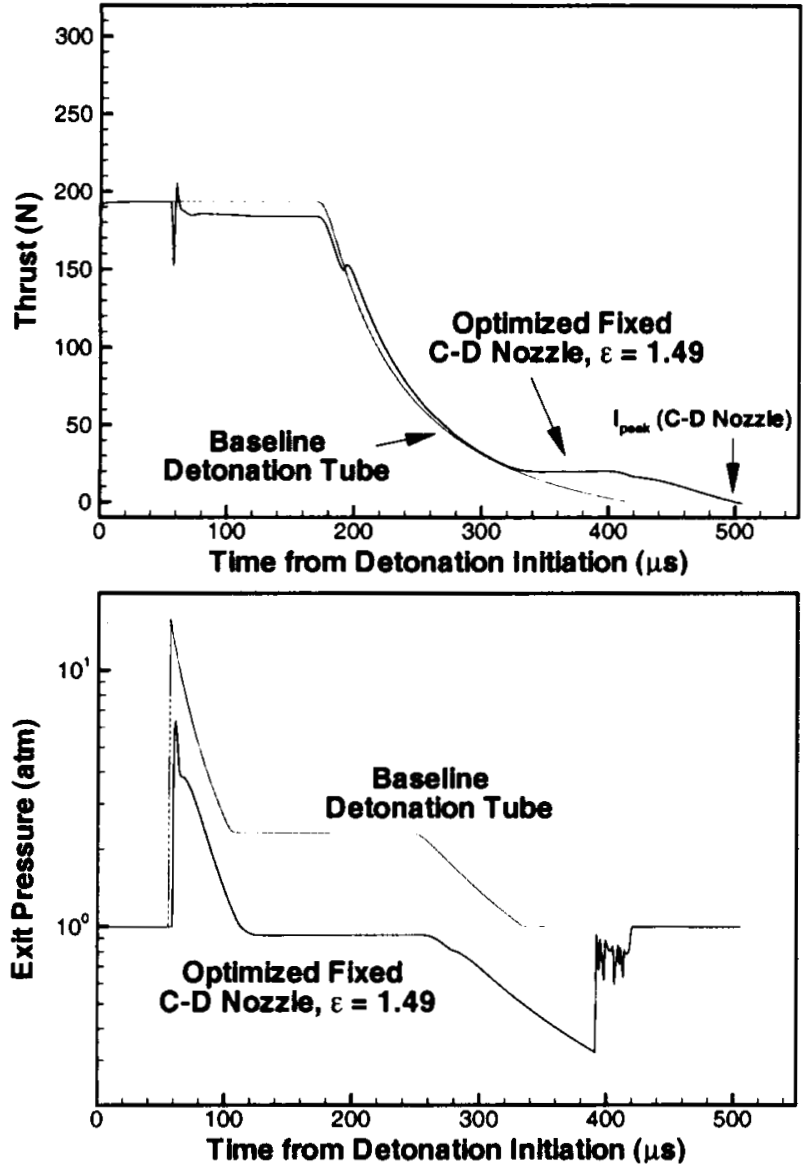

Fig. 4 Comparison of thrust (upper panel) and exit pressure (lower panel) histories for PDRE systems at a blowdown pressure ratio of 1. Propellant mixture: stoichiometric $\mathrm{H}_{2}-\mathrm{O}_{2}$. Propellant initial conditions: $P_{\text {initial }}=1 \mathrm{~atm}, T_{\text {initial }}=300 \mathrm{~K}$.

an optimized fixed C-D nozzle can provide only marginal benefit ( $\sim 3 \%$ additional specific impulse) over the baseline detonation tube at a blowdown pressure ratio of 1 .

In contrast, substantial benefits can be realized from a C$\mathrm{D}$ nozzle at larger blowdown pressure ratios. The optimum nozzle exit diameter for the model PDRE system at a blowdown pressure ratio of 1000 is found to be $18.2 \pm 0.2 \mathrm{~cm}$. This corresponds to an expansion ratio of $102 \pm 2$. The mixture-based specific impulse of the optimized C-D system is $400.11 \mathrm{~s}$, a significant improvement over the $262.04 \mathrm{~s}$ provided by the baseline detonation tube at this pressure ratio. A comparison between the single-shot thrust history for the optimized converging-diverging nozzle and the baseline detonation tube is shown in the upper panel of Fig. 5. The corresponding exit pressure history is shown in the lower panel of the figure. As is evident from the figure, the blowdown process (to $0.001 \mathrm{~atm}$ ) is considerably longer in this case than in the previous one. Note that the optimized $\mathrm{C}$ D nozzle provides considerably higher thrust, compared to the baseline tube, throughout the blowdown history. This is due to the familiar result from classical compressible flow that the best performance from a supersonic rocket nozzle is obtained when the pressure at the exit plane is expanded to the ambient value. As may be seen in the exit pres-
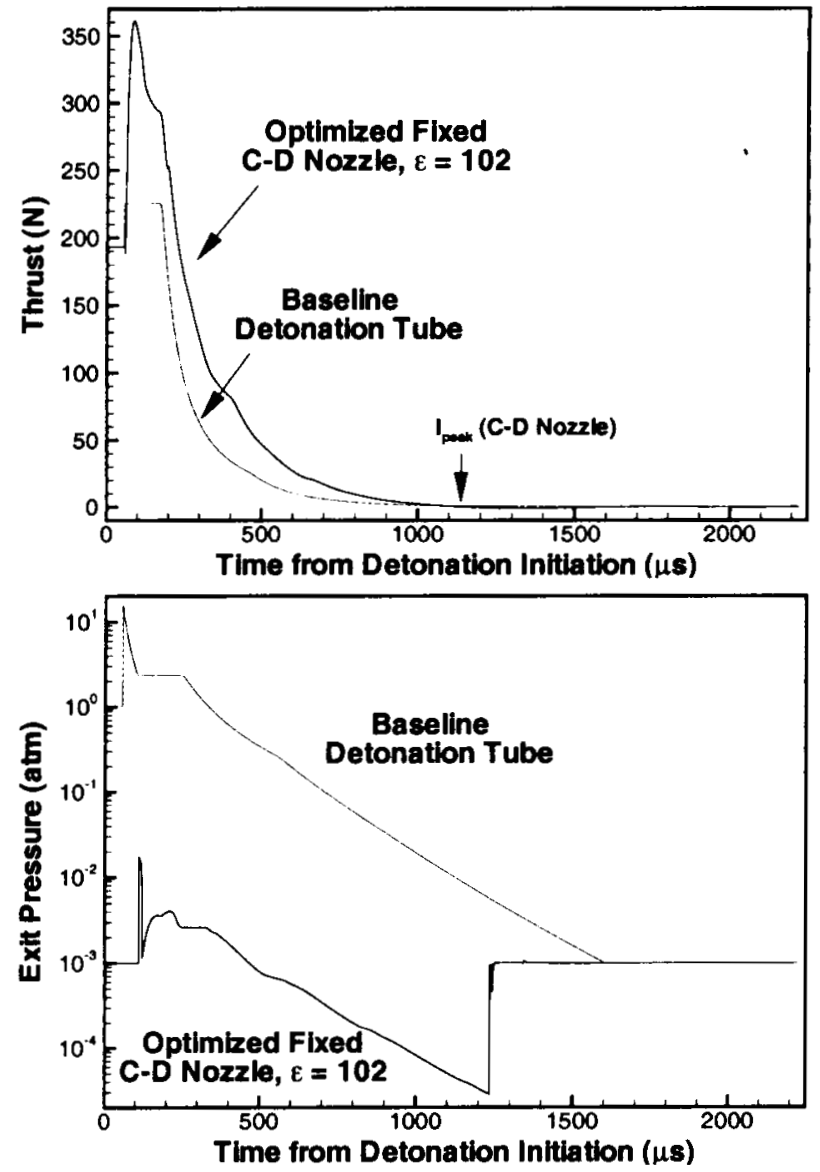

Fig. 5 Comparison of thrust (upper panel) and exit pressure (lower panel) histories for PDRE systems at a blowdown pressure ratio of 1090. Propellant mixture: stoichiometric $\mathrm{H}_{2}-\mathrm{O}_{2}$. Propellant initial conditions: $P_{\text {initial }}=1 \mathrm{~atm}, T_{\text {initial }}=300 \mathrm{~K}$.

sure history, the C-D nozzle expands the exhaust flow by roughly three orders of magnitude compared to the baseline tube. Thus, while the fixed nozzle cannot dynamically adapt to provide optimum expansion throughout the entire blowdown process, the resultant performance gain is still quite significant $(\sim 53 \%)$. It is worth while to note that the peak impulse (as shown by the marker in the upper panel) is reached by $1141 \mu \mathrm{s}$, roughly half the entire blowdown time. After this point, the thrust oscillates both slightly above and below zero, leading to a small loss in impulse by the end of the blowdown process $(\sim 0.4 \%)$. This suggests that, in an actual cyclic PDRE system using this geometry, purging and propellant refill should begin much earlier than allowing the end-wall pressure to decay to the ambient pressure value.

\section{Equivalent SSRE Model}

It is instructive to compare the specific impulse of both PDRE systems with a SSRE under equivalent modeling assumptions. While frozen and equilibrium rocket performance calculations can be readily obtained from the CEA code, these results are not directly comparable to the finiterate chemistry model used in the current PDRE code. The primary concern is the tendency for chemistry to slow down 
in real nozzle systems as the temperature and pressure are reduced in the expansion process. Thus, it is best to compare the finite-rate PDRE systems with a finite-rate SSRE model. This SSRE CFD model is heavily derived from the PDRE code. The $16 \mathrm{~cm}$ detonation tube is replaced with a thrust chamber $0.1 \mathrm{~cm}$ in length, and $3.6 \mathrm{~cm}$ in diameter (Fig. 6). This thrust chamber then converges at a constant $14^{\circ}$ angle to a throat $1.8 \mathrm{~cm}$ in diameter. After the throat, the nozzle again expands at $14^{\circ}$ to the exit diameter specified by the user. The throat diameter and nozzle expansion rate are thus identical in both the PDRE and SSRE models. The equilibrium temperature, pressure and composition of stoichiometric $\mathrm{H}_{2}-\mathrm{O}_{2}$, burned at constant pressure and enthalpy from initial conditions of $300 \mathrm{~K}$ and $1 \mathrm{~atm}$, are fed as a constant enthalpy reservoir inflow boundary condition into the domain.

Similar to the PDRE nozzle optimization study, the finite-rate SSRE CFD model is run at various expansion ratios until the optimum specific impulse is obtained for a given pressure ratio. However, as the solution converges to steady-state in this model, flowfield information from the highest pressure ratio case can be used to guide area selection at the lower pressure ratios. In each case, the SSRE model is run until the solution converges. In general, the specific impulse and optimum expansion ratio using finite-rate chemistry are slightly larger than that for frozen chemistry. Additionally, if the chemistry is frozen in the SSRE CFD model, there is excellent agreement (to within $0.3 \%$ in specific impulse) with the frozen-chemistry predictions of CEA.

\section{Performance Comparison}

The mixture-based specific impulse for the baseline detonation tube, the optimized fixed C-D nozzle PDRE system, and the equivalent optimized SSRE system is plotted for blowdown pressure ratios of $1,2,5,10,20,50,100$, 200,500 and 1000 in the upper panel of Fig. 7. The corresponding area ratios for the C-D systems are shown in the lower panel of the figure. As would be expected from the example results discussed previously, the relative gain from the C-D nozzle systems becomes more pronounced at higher pressure ratios. It is interesting to note that that both the baseline and C-D nozzle PDRE systems outperform a SSRE at pressure ratios below $\sim 7$. This is due to the fact that the relative gain from the extra pressurization provided by the detonation process is greatest in this regime. Thus, the PDRE may have considerable theoreti-

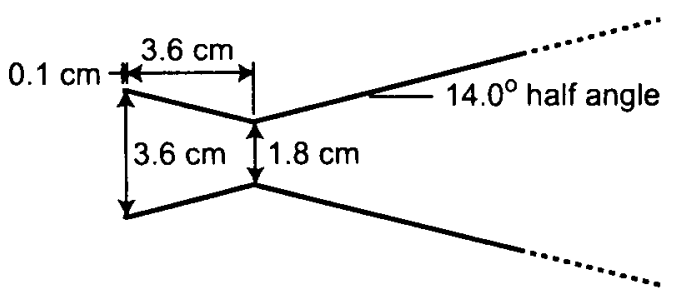

Fig. 6 Schematic of SSRE geometry studied.
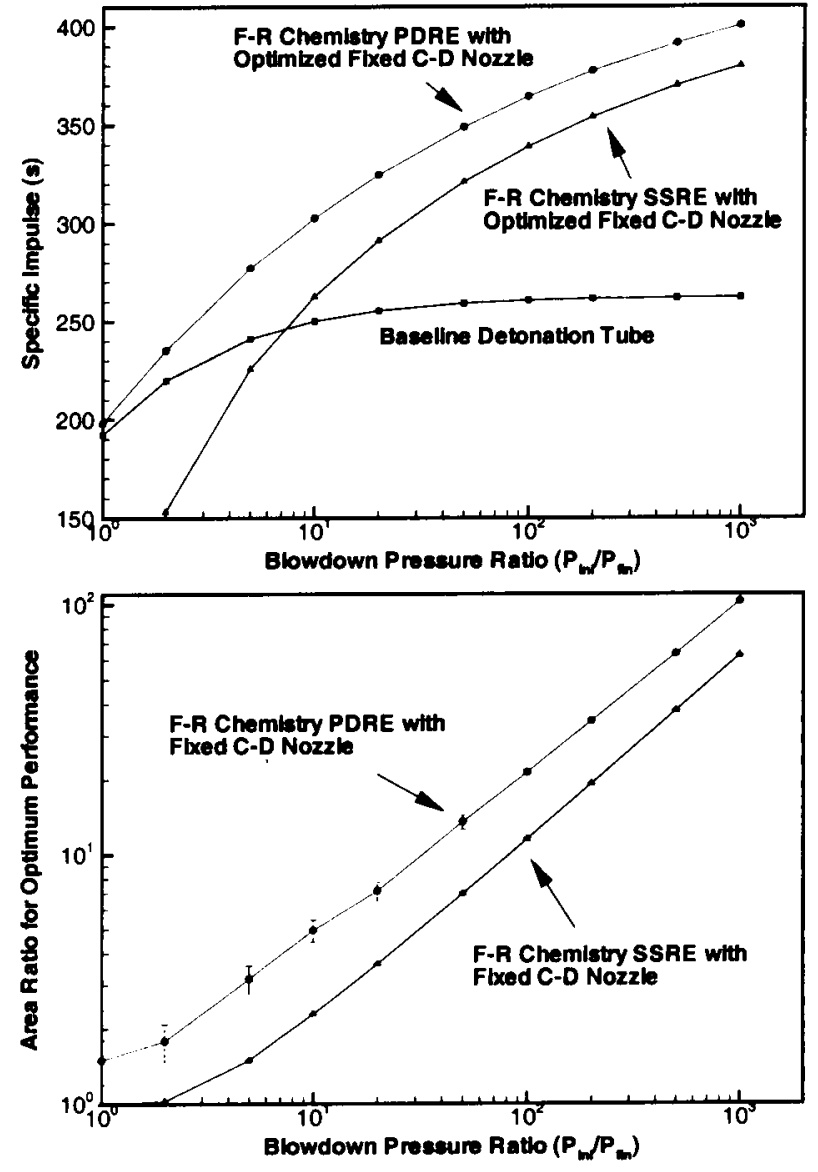

Fig. 7 Performance (upper panel) and area ratio (lower panel) comparison of various pulse detonation and steadystate rocket devices. All results obtained using finite-rate quasi 1-D CFD calculations. The final blowdown pressure at the end-wall in the PDRE is equal to the ambient pressure $\left(P_{\text {final }}=P_{\text {ambient }}\right)$. Propellant mixture: stoichiometric $\mathrm{H}_{2}-\mathrm{O}_{2}$. Propellant initial conditions: $P_{\text {initial }}=1 \mathrm{~atm}, T_{\text {initial }}=300 \mathrm{~K}$.

cal potential for rocket-type applications when the pressure of the ambient environment is high. Additionally, at higher blowdown pressure ratios, a PDRE with an optimized, fixed C-D nozzle has a greater specific impulse than an equivalent SSRE. These results indicates that a PDRE equipped with a fixed C-D nozzle, though obviously not as efficient at generating thrust as a (theoretical) dynamically adaptive nozzle, can still yield performance superior to an equivalent SSRE over a wide range of pressure ratios. Note, however, that this performance gain becomes relatively smaller at higher pressure ratios.

\section{Summary and Future Work}

A quasi 1-D, finite-rate chemistry CFD model for studying PDRE gasdynamics and performance is described and implemented. The performance of a simple fixed, but optimized, converging-diverging nozzle design is compared with a baseline detonation tube over a range of blowdown pressure ratios from 1-1000. The results demonstrate that even relatively simple fixed nozzle designs can make significant improvements in PDRE performance at high pres- 
sure ratios. Additionally, as practical systems would likely employ cryogenically-stored, fuel-rich propellant mixtures, work is planned to repeat the optimization study for a range of stoichiometries and initial temperatures. Work is also underway to implement an ethylene chemistry model for comparison with experimental results from the literature.

\section{References}

${ }^{1}$ Bratkovich, T. E., Aamio, M. J., Williams, J. T., and Bussing, T. R. A., "An Introduction to Pulse Detonation Rocket Engines," AIAA paper no. 97-2742 at the 33rd AIAA/ASME/SAE/ASEE Joint Propulsion Conference and Exhibit, Seattle, WA, July 6-9, 1997.

${ }^{2}$ Kailasanath, K., "A review of PDE Research - Performance Estimates," AIAA paper no. 2001-0474 at the 39th AIAA Aerospace Sciences Meeting, Reno, NV, January 8-11, 2001.

${ }^{3}$ Morris, C. I., "Simplified Analysis of Pulse Detonation Rocket Engine Blowdown Gasdynamics and Performance," AIAA paper no. 20023715 at the 38th AIAA/ASME/SAE/ASEE Joint Propulsion Conference and Exhibit, Indianapolis, IN, July 7-10, 2002.

${ }^{4}$ McBride, B. J., Gordon, S., and Reno, M. A., "Coefficients for Calculating Thermodynamic and Transport properties of Individual Species," Tech. Rep. TM 4513, NASA, 1993.

${ }^{5}$ Petersen, E. L. and Hanson, R. K., "Reduced Kinetic Mechanisms for Ram Accelerator Combustion," Journal of Propulsion and Power, Vol. 15, No. 4, 1999, pp. 591-600.

${ }^{6}$ Kee, R. J., Rupley, F. M., and Miller, J. A., "Chemkin-II: A FORTRAN Chemical Kinetics Package for the Analysis of Gas-Phase Chemical Kinetics," Tech. Rep. SAND89-8009, Sandia National Laboratories, 1989.

${ }^{7}$ Troe, J., "Predictive Possibilites of Unimolecular Rate Theory," Journal of Physical Chemistry, Vol. 83, No. 1, 1979, pp. 114-126.

${ }^{8}$ Strang, G., "On the Construction and Comparison of Difference Schemes," SIAM Journal of Numerical Analysis, Vol. 5, 1968, pp. 506517.

${ }^{9}$ Oran, E. S. and Boris, J. P., Numerical Simulation of Reactive Flow, Cambridge University Press, 2nd ed., 2001, pp. 408-411.

${ }^{10}$ Yee, H. C., "A Class of High-Resolution Explicit and Implicit Shock-Capturing Methods," Tech. Rep. TM 101088, NASA, 1989.

${ }^{11}$ Grossman, B. and Cinnella, P., "Flux-Split Algorithms for Flows with Non-equilibrium Chemistry and Vibrational Relaxation," Journal of Computational Physics, Vol. 88, 1990, pp. 131-168.

${ }^{12}$ Larrouturou, B., "How to Preserve the Mass Fractions Positivity when Computing Compressible Multi-component Flows," Journal of Computational Physics, Vol. 95, 1991, pp. 59-84.

${ }^{13}$ Mott, D. R., New Quasi-Steady-State and Partial-Equilibrium Methods for Integrating Chemically Rearting Systems, Ph.D. thesis, University of Michigan, 2000.

${ }^{14}$ Poinsot, T. J. and Lele, S. K., "Boundary Conditions for Direct Simulations of Compressible Viscous Flows," Journal of Computational Physics, Vol. 101, 1992, pp. 104-129.

${ }^{15}$ Mohanraj, R. and Merkle, C. L., "A Numerical Study of Pulse Detonation Engine Performance," AIAA paper no. 2000-0315 at the 38th AIAA Aerospace Sciences Meeting and Exhibit, Reno, NV, January 10$13,2000$.

${ }^{16}$ Gordon, S. and McBride, B. J., "Computer Program for Calculation of Complex Chemical Equilibrium Compositions and Applications," NASA Reference Publication 1311, 1994. 